\title{
Le gène de la chorée de Huntington : la fin d'une longue traque
}

La chorée de Huntington est une maladie neurodégénérative transmise selon le mode autosomique dominant et affectant un individu sur 10000 dans la plupart des populations européennes. Le locus portant le défaut moléculaire responsable de cette affection a été localisé en 1983 par J. Gusella sur le bras court du chromosome 4 (4p16.3) [1]. Par des études de déséquilibre de liaison dans différentes populations et d'événements de recombinaison observés chez des malades, deux régions candidates susceptibles de contenir le gène avaient été mises en évidence, toutes deux situées en 4p16.3 mais séparées d'environ 1.5 mégabases. Finalement, un fragment de $600 \mathrm{~kb}$ situé dans la région proximale entre les marqueurs D4S180 et D4S182 semblait être la région candidate la plus vraisemblable. Ce gène vient d'être identifié grâce à la collaboration de six groupes de recherche américains et anglais (Gusella, Lehrach, Housman, Wasmuth, Collins, Harper) [2]. L'ADNc de ce gène a été cloné par la méthode des exons piégés (exon trapping). Toute une séric de cosmides chevauchants couvrant la région candidate de $600 \mathrm{~kb}$ ont été isolés. Les insertions de ces vecteurs ont été isolées et clivées par des enzymes de restriction. Ces fragments ont ensuite été insérés dans un vecteur bordé par deux exons synthétiques associés l'un à un site donneur et l'autre à un site accepteur d'épissage. Après transfection de cellules en culture par ces cosmides, les ARN cellulaires sont utilisés pour réaliser des amplifications par PCR en se servant d'amorces spécifiques des deux exons artificiels. Tout exon interne sera épissé aux deux exons artificiels et amplifié par PCR [3]. Ainsi fut notamment obtenu un exon contenant un triplet CAG répété qui fut utilisé pour cribler une banque d'ADNc de cortex frontal humain. Plusieurs clones furent obtenus correspondant à un $\mathrm{ARNm}$ de 10 à $11 \mathrm{~kb}$ dénommé IT16. Cet ARNm est exprimé dans plusieurs régions du cerveau, mais aussi dans d'autres tissus tels que le foie, le rein, la rate et le muscle. Il code pour une protéine d'environ $340 \mathrm{kDa}$ de fonction inconnue. Le cadre de lecture d'IT16 contient une répétition du trinucléotide CAG. Le nombre de copies CAG varie de 11 à 34 dans les allèles normaux. Le nombre de triplets CAG a été recherché chez 75 malades provenant d'origines ethniques différentes. Chez tous ces patients, l'allèle muté contient un nombre de répétitions situé entre 42 et 86 . Il est connu que le taux de mutation spontanée dans la chorée de Huntington est très faible. Deux cas sporadiques ont été étudiés par les auteurs. Dans les deux cas, une augmentation du nombre de triplets (44 et 46 copies) a été observé chez les malades alors que l'allèle parental correspondant est associé à un nombre de triplets situés à la limite supérieure de la normale (36 copies). De plus, dans ces deux cas, l'haplotype de l'allèle muté observé correspond à l'haplotype le plus fréquemment observé chez les malades. Cela suggère qu'il existe peut-être un haplotype particulier prédisposant à l'amplification de triplets, ce qui a été déjà observé dans la dystrophie myotonique de Steinert $(\mathrm{m} / \mathrm{s}$ $n^{\circ} 3$, vol. 8, p. 249). La chorée de Huntington est donc chronologiquement la quatrième maladie génétique résultant d'une expansion de triplets, les trois précédentes étant le syndrome de l'X fragile, la dystrophie myotonique de Steinert et le syndrome de Kennedy ( $m / s n^{\circ} 9$, vol. 8 , p. 983). Comme dans ces trois affections le nombre de répétitions du triplet observées dans la chorée de Huntington est instable et subit une expansion au cours des générations. Cependant, cette expansion semble être limitée, car, dans les 75 chromosomes mutés étudiés, le nombre de répétitions ne dépasse pas 86 (2 à 3 fois plus que la normale). A ce titre, le mécanisme moléculaire conduisant à la chorée de Huntington semble plus ressembler au syndrome de Kennedy $\left(\mathrm{m} / \mathrm{s} n^{\circ} 7\right.$, vol. 7, p. 752) qu'aux deux autres maladies où le nombre de répétitions peut subir des expansions beaucoup plus grandes (10 fois et plus). Un autre point commun avec le syndrome de Kennedy est la nature du triplet, CAG, présent dans les deux cas dans la partie codante du gène. Un autre fait intéressant est que le nombre de répétitions du triplet semble être corrélé à l'âge d'apparition des premiers symptômes. Les nombres de répćtition les plus élevés étant retrouvés dans les formes juvéniles où la transmission paternelle est la plus fréquente.

Autre question posée : comment cette mutation peut-elle conduire à un phénotype dominant? Les auteurs ont observés que l'ARNm IT16 est présent chez les patients hétérozygotes et homozygotes. Cela suggère que l'amplification de triplet ne provoque pas une inactivation du gène. Puisqu'il n'est pas possible cliniquement de distinguer les malades homozygotes des hétérozygotes, les auteurs en arrivent à la conclusion que la symptomatologie est due à un gain de fonction provoquée par la mutation qui se mani- feste, soit au niveau de l'ARNm, soit au niveau de la protéine. Si l'ARNm est traduit, les conséquences sur la protéine seront dramatiques par la présence d'une séquence poly-gln près de l'extrémité $\mathrm{N}$ terminale. Il est possible aussi que le codon d'initiation soit présent en 3' de l'expansion de triplet, auquel cas la mutation se trouve dans la partie 5' non codante et peut affecter la traduction. Dans ce cas, cependant, la mutation entraînerait plutôt une perte de fonction et les homozygotes devraient être plus atteints que les hétérozygotes. Une autre hypothèse est que l'expansion du triplet modifierait le site d'initiation de la traduction, entraînant l'apparition d'une protéine tronquée. Il n'est actuellement pas possible d'exclure l'influence de cette mutation sur l'expression d'un autre gène. Simultanćment à la parution de l'article de Cell, une équipe canadienne a rapporté que chez 250 malades analysés, ils en avaient détecté deux avec une insertion de $331 \mathrm{pb}$ correspondant à la rétrotransposition d'un élément mobile de la famille Alu [4]. L'analyse de la carte de restriction a montrć que deux gènes sont présents dans la région contenant l'insertion Alu : l' $\alpha$-adducine $\left(m / s \quad n^{\circ} 1\right.$, vol. 9, p. 103) et un transcrit de $12 \mathrm{~kb}$ qui correspond probablement à l'ARNm IT16 cité dans l'article précédent. Ces deux gènes sembleraient être imbriqués l'un dans l'autre, ce qui est un cas très rarement rencontré chez l'homme. Il n'est cependant pas confirmé que l'insertion de la séquence Alu soit responsable de l'affection. De plus, les auteurs n'ont pas étudié chez ces deux malades le nombre de triplets CAG

C.D.

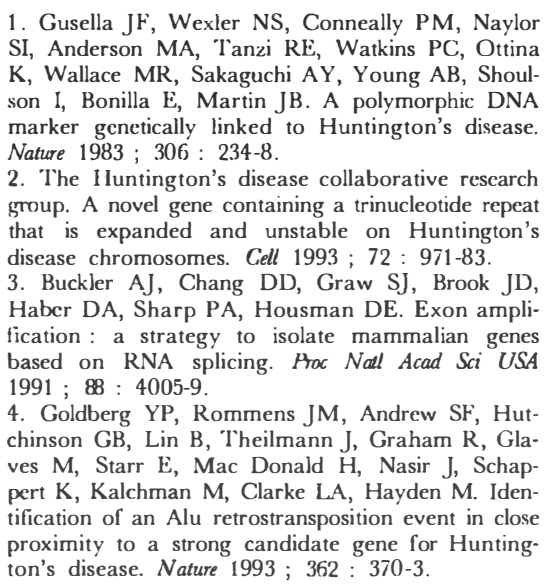

1. Gusella JF, Wexler NS, Conneally PM, Naylor SI, Anderson MA, Tanzi RE, Watkins PC, Ottina son I, Bonilla E, Martin JB. A polymorphic DNA marker genetically linked to Huntington's disease. Nature $1983 ; 306$ : 234-8.

2. The lluntington's disease collaborative research group. A novel gene containing a trinucleotide repeat disease chromosomes. Cell 1993 ; 72 : 971-83

3. Buckler AJ, Chang DD, Graw SJ, Brook JD, Haber DA, Sharp PA, Housman DE. Exon amplibased on RNA splicing. Hroc Nat Acad Sci USA $1991 ; 88$ : 4005-9.

4. Goldberg YP, Rommens JM, Andrew SF, Hutchinson GB, Lin B, Theilmann J, Graham R, Glaproximity to a strong candidate gene for Hunting ton's disease. Nature $1993 ; 362: 370-3$ 